Pesq. Vet. Bras. 30(7):593-604, julho 2010

\title{
Imagem por ressonância magnética na investigação da cabeça de cães ${ }^{1}$
}

\author{
Maria Cristina F.N.S. Hage ${ }^{2^{*}}$, Masao Iwasaki ${ }^{3}$, Said R. Rabbani ${ }^{4}$, Lilian \\ Kamikawa ${ }^{3}$, Hernan J.R. Cervantes ${ }^{4}$, Pedro P. Bombonato ${ }^{3}$, Franklin A. \\ Sterman $^{3}$ e Maria C.G. Otaduy ${ }^{5}$
}

\begin{abstract}
Hage M.C.F.N.S., Iwasaki M., Rabbani S.R., Kamikawa L., Cervantes H.J.R., Bombonato P.P., Sterman F.A. \& Otaduy M.C.G. 2010. [Magnetic resonance imaging in the investigation of canine heads.] Imagem por ressonância magnética na investigação da cabeça de cães. Pesquisa Veterinária Brasileira 30(7):593-604. Departamento de Veterinária, Universidade Federal de Viçosa, Av. P.H. Rolfs s/n, Viçosa, MG 36570-000, Brazil. E-mail: crishage@ ufv.br

Magnetic resonance imaging (MRI) is the most sensitive method of diagnostic imaging to evaluate soft tissues, specially the brain, however it is expensive. The method is based on the nuclear magnetic resonance phenomenon that occurs when atomic nucleus with magnetic proprieties in the body are submitted to a strong magnetic field, and excited with radio frequency generating a radio frequency signal captured by a receptive antenna. The signal is processed by Fourier Transform for the image formation. This study had the objective to obtain 10 complete exams of heads in cadavers of normal dogs to MRI and to make an Atlas of head structures. The images were obtained with a magnetic resonance unit Gyroscan S15/HP Philips using a magnetic field of 1,5Tesla. The cadavers were positioned with the head into a human head coil and submitted to sagittal slices used to plan transverse and dorsal slices in T1, T2 and DP spin-echo sequences. In T1 we adjusted $T R=400 \mathrm{~ms}$ and $T E=30 \mathrm{~ms}$, in T2 TR=2000ms and TE $=80 \mathrm{~ms}$ and in DP TR=2000ms and $\mathrm{TE}=30 \mathrm{~ms}$. The slice thickness was $4 \mathrm{~mm}$, the number of averages 2, the matrix $256 \times 256$, the factor 1,0 and the field of view $14 \mathrm{~cm}$. The duration of the complete exam of the head was 74,5 minutes. The images obtained with the described sequences and with the human head coil was of good quality. In T1 fat was hyperintense and fluid was hypointense. In T2 fat was less hyperintense and fluid was hyperintense. The cortical bone and the air were hypointense in all sequences used because of the low proton density. The DP sequence showed the best contrast between white and gray matter when compared with T2 and T1 sequences. Distinction of cerebral sulcus and gyrus was possible because T2 showed the cerebrospinal fluid. The identification of bone structures that compound the region, muscles, main venous and arterial vessels and structures of the central nervous system, besides elements of the digestory and respiratory systems and structures of the eyes among others was possible through contrast obtained with MRI. In this study the MRI acquired in T1, DP and T2 were complementary for the anatomic study of the head and been able to demonstrate the structures of the canine head with rich anatomic details. The time used to
\end{abstract}

\footnotetext{
${ }^{1}$ Recebido em 29 de novembro de 2009.

Aceito para publicação em 24 de fevereiro de 2010.

2 Diagnóstico por Imagem, Departamento de Veterinária (DVT), Universidade Federal de Viçosa (UFV), Av. P.H. Rolfs s/n, Campus Universitário, Viçosa, MG 36570-000, Brasil. "Autor para correspondência: crishage@ufv.br

${ }^{3}$ Departamento de Cirurgia, Faculdade de Medicina Veterinária e
}

\footnotetext{
Zootecnia (FMVZ), Universidade de São Paulo (USP), Av. Prof. Dr. Orlando Marques de Paiva 87, Cidade Universitária, São Paulo, SP 05508-270, Brasil.

${ }^{4}$ Instituto de Física, USP, Rua do Matão, Travessa R, 187, Cidade Universitária, São Paulo, SP 05508-090.

${ }^{5}$ Departamento de Radiologia, Faculdade de Medicina, USP, Av. Dr. Enéas de Carvalho Aguiar 255, São Paulo, SP 05403-001.
} 
do the complete exam of the head is compatible with the use in live animals since properly anesthetized and controlled. We had opened a way for the study of live animals and for the beginning of disease investigation, mainly that of neurologic origin because this technique is excellent for brain visualization.

INDEX TERMS: MRI, brain, canine species, animal anatomy.

RESUMO.- A imagem por ressonância magnética (IRM) é o método de diagnóstico por imagem não invasivo mais sensível para avaliar as partes moles, particularmente o encéfalo, porém trata-se de uma técnica onerosa. $O$ método fundamenta-se no fenômeno da ressonância magnética nuclear que ocorre quando núcleos atômicos com propriedades magnéticas presentes no corpo são submetidos a um campo magnético intenso, sendo posteriormente excitados por energia de radiofrequência e gerando, por sua vez, um sinal de onda de radiofrequência capaz de ser captado por uma antena receptora, passando por um processo matemático, chamado Transformada de Fourier, para posterior formação da imagem. Esse estudo objetivou realizar 10 exames completos da cabeça em cadáveres de cães normais à IRM e confeccionar um Atlas com as estruturas identificadas. As imagens foram adquiridas em um aparelho de ressonância magnética Gyroscan S15/HP Philips com campo magnético de 1,5Tesla. Os cadáveres foram posicionados com a cabeça no interior de uma bobina de cabeça humana e foram submetidos a cortes iniciais sagitais a partir de onde se planejou os cortes transversais e dorsais nas sequências de pulso spin-eco T1, T2 e DP. Em T1 utilizou-se TR=400ms e TE $=30 \mathrm{~ms}$, T2 utilizou-se TR=2000ms e TE $=80 \mathrm{~ms}$ e na DP utilizou-se $T R=2000 \mathrm{~ms}$ e TE$=30 \mathrm{~ms}$. A espessura do corte foi de $4 \mathrm{~mm}$, o número de médias foi igual a 2, a matriz foi de 256x256, o fator foi igual a 1,0 e o campo de visão foi de $14 \mathrm{~cm}$. A duração do exame completo da cabeça foi de 74,5 minutos. As imagens obtidas com as sequências utilizadas e com a bobina de cabeça humana foram de boa qualidade. Em T1 a gordura tornou-se hiperintensa e o líquido hipointenso. Em T2 a gordura ficou menos hiperintensa e o líquido hiperintenso. A cortical óssea e o ar foram hipointensos em todas as sequências utilizadas devido a baixa densidade de prótons. A sequência DP mostrou o melhor contraste entre a substância branca e cinzenta quando comparada a T2 e a T1. T2 evidenciou o líquido cefalorraquidiano tornando possível a distinção dos sulcos e giros cerebrais. Através do exame de IRM foi possível, pelo contraste, identificar as estruturas ósseas componentes da arquitetura da região, músculos, grandes vasos venosos e arteriais e estruturas do sistema nervoso central, além de elementos do sistema digestório, respiratório e estruturas dos olhos entre outras. Nesse estudo as IRM adquiridas nas sequências T1, DP e T2 foram complementares para o estudo dos aspectos anatômicos da cabeça de cães demonstrando-os com riqueza de detalhes. O tempo requerido para o exame completo da cabeça é compátivel para uso em animais vivos desde que devidamente anestesiados e controlados. Os resultados obtidos por esse trabalho abrem caminho em nosso meio, para o estudo de animais vivos e para o início da investigação de doenças, principalmente as de origem neurológica, visto ser esta técnica excelente para a visibilização do encéfalo.

TERMOS DE INDEXAÇÃO: IRM, encéfalo, espécie canina, anatomia animal.

\section{INTRODUÇÃO}

A geração de IRM revolucionou a medicina na obtenção de imagens. A ressonância magnética supera por ampla margem a tomografia computadorizada em sua capacidade de distinguir diferenças sutis nos limites dos tecidos moles. $\mathrm{O}$ contraste nas imagens se baseia na diferença de intensidade do sinal em áreas de estruturas ou de composição diferentes. A capacidade de obtenção de imagens multiplanares e a ausência de radiação ionizante tornaram a ressonância magnética a técnica de geração de imagens mais adequada para muitas doenças, em especial as do sistema nervoso central (SNC) por causa do seu abundante conteúdo hídrico, portanto, rico em prótons de hidrogênio, responsáveis pelo sinal gerado durante a obtenção de imagens (Lufkin 1999, Hilário et al. 2000, Amaral et al. 2003, Mullins 2006, Hage \& Iwasaki 2009).

As indicações clínicas do exame de IRM no encéfalo são as anormalidades congênitas, os traumas do SNC, os processos infecciosos, a doença vascular cerebral (Platt \& Garosi 2003, Desal et al. 2004, Mullins 2006) e a hidrocefalia (Assheuer \& Sager 1997).

De todas as aplicações da IRM, o estudo dos tumores cerebrais parece a mais instigante, pelas inovações de tratamento que podem ser instituidas à partir da imagem (Fonseca-Pinto et al. 2008). A determinação da origem, localização, forma e padrão de crescimento são características que podem ser obtidas com as IRM. A intensidade do sinal e o padrão de realce do contraste permitem diferenciações adicionais (Kraft et al. 1997).

Diante dessas vantagens incontestáveis existe grande interesse por parte dos médicos veterinários radiologistas na utilização dessa técnica, porém, o seu alto custo dificulta a disponibilidade do equipamento. Outros fatores importantes a serem consolidados para quem almeja interagir com essa metodologia de imagem, são a compreensão da formação da imagem e o conhecimento da anatomia da cabeça em cortes seccionais. Recentemente foi publicada uma revisão da literatura sobre os princípios básicos dessa modalidade de imagem de uma forma assimilável para não físicos (Hage \& Iwasaki 2009).

O importante desafio sobre o conhecimento da anatomia seccional levou a produção de vários artigos científicos sobre o assunto, com a finalidade de confeccionar Atlas de diferentes espécies. Em relação ao cão foram elaborados Atlas sobre a anatomia do cérebro (Kraft et al. 
1989, Assheuer \& Sager 1997), do olho e da órbita (Morgan et al. 1994), dos linfonodos da cabeça e pescoço (Kneissl \& Probst, 2006), das cavidades nasais e seios paranasais (De Rycke et al. 2003), e da laringe (Vázquez et al. 1998), entre outros. Foram também estudados o cérebro de gatos (Hudson et al. 1995) e de cavalos (Arencibia et al. 2001, Chaffin et al. 1997). Curiosamente há relatos de Atlas de anatomia cranioencefálica do camelo (Arencibia et al. 2005) e do golfinho (Marino et al, 2001). Na medicina humana, apesar de todo o avanço na aplicação dessa modalidade de imagem, ainda continuam os esforços sobre a busca do reconhecimento da anatomia detalhada, por ser um requerimento imprescindível para o reconhecimento e interpretação das alterações (Blink et al. 2009, Hermie et al, 2009, Oishi, 2008).

Corroborando com os estudos anteriores, objetivou-se realizar 10 exames completos da cabeça em cadáveres de cães normais à IRM, com a finalidade de demonstrar que os resultados são reproduzíveis e confeccionar um Atlas com imagens por ressonância magnética, identificando as estruturas da cabeça nas sequências spin-eco relaxação T1 (T1), densidade de prótons (DP) e relaxação T2 (T2), explicando as diferenças das tonalidades de cinza.

\section{MATERIAL E MÉTODOS}

Aparelho. As imagens foram adquiridas utilizando o Tomógrafo de Ressonância Magnética Nuclear de corpo inteiro da Marca Philips, Gyroscan S15, com potência de campo magnético de 1,5 Tesla do Instituto de Física da Universidade de São Paulo.

Animais. O estudo foi realizado em dez cadáveres de cães, selecionados como clinicamente normais previamente a eutanásia, pesando entre 10 e $30 \mathrm{~kg}$, provenientes do Centro de Controle de Zoonoses de São Paulo. O estudo foi aprovado pela Comissão de Bioética da Faculdade de Medicina Veterinária e Zootecnia da Universidade de São Paulo, sob o protocolo número 436/2004.

Protocolo. As imagens foram adquiridas utilizando bobina para cabeça humana e sequência de pulso spin-eco ponderada em T1, DP e T2.

Para série localizadora sagital utilizou-se a seguinte programação: número de ecos: 1; tempo de repetição: 457 mseg; tempo de eco: 15mseg; fator da fatia: 1; número de fatias: 5; espessura da fatia: $4 \mathrm{~mm}$; número de médias: 1 ; campo de visão: $22 \mathrm{~cm}$.

Para a série transversal e dorsal em DP e T2 utilizou-se: número de ecos: 2; tempo de repetição: 2000 mseg; tempo de eco: $30 \mathrm{mseg}$ (DP) e $80 \mathrm{mseg}$ (T2); fator da fatia: 1; número de fatias: 25 - 30; espessura da fatia: $4 \mathrm{~mm}$; número de médias: 2 ; campo de visão: $14 \mathrm{~cm}$.

Para a série T1 transversal e dorsal utilizou-se: número de ecos: 1; tempo de repetição: 400mseg; tempo de eco: 30mseg; fator da fatia: 1; número de fatias: 25-30; espessura da fatia: $4 \mathrm{~mm}$; número de médias: 2; campo de visão: $14 \mathrm{~cm}$.

Método. Os cadáveres foram posicionados sobre uma prancha de madeira em decúbito dorsal, as orelhas foram presas à cabeça com a ajuda de fita adesiva e, esta presa à madeira pela região rostral do focinho. Os membros torácicos foram imobilizados paralelos um ao outro e posicionados sobre o tórax por meio de fita adesiva e fitilho. Os membros pélvicos foram imobilizados em extensão e presos na prancha com fita adesiva e fitilho. Posteriormente, o animal foi posicionado na luz orientadora e introduzindo no aparelho.

Manipulação das imagens. As imagens obtidas foram gravadas em "compact disk" sendo cada corte numerado sequencialmente com terminação ani (específica para imagens). Tais imagens foram tratadas uma a uma através do programa MRIcro ${ }^{\circledR}$ disponível no site www.mricro.com. Após abertas as imagens foram copiadas uma a uma e coladas no programa PrestolmageFolio ${ }^{\circledR}$ integrante do Scanner Genius ColorPageHR6Xslim ${ }^{\circledR}$, onde foi realizada a inscrição de setas e números. Após, foram inseridas no programa Power Point ${ }^{\circledR}$ onde foram recortadas e transformadas para escala de cinza.

Confecção do Atlas. Entre os 10 exames realizados foi selecionado um para a confecção do Atlas com as estruturas identificadas nas IRM. Esse exame foi comparado com os demais 9 exames com a finalidade de demonstrar sua repetibilidade. As imagens do exame escolhido foram organizadas com as três sequências utilizadas (T1, DP e T2), para fins de comparação e as estruturas anatômicas foram identificadas e legendadas, baseadas nos Atlas e textos de anatomia veterinária (Kraft et al. 1989, Feeney et al. 1991, Assheuer \& Sager 1997, Schaller 1999). Os nomes das estruturas seguem as normas estabelecidas pela Nomenclatura Anatômica Veterinária llustrada (Schaller 1999). Cada corte contém a imagem sagital demonstrando a orientação e a localização do corte.

\section{RESULTADOS E DISCUSSÃO}

A aquisição das imagens sagitais demorou aproximadamente 2,5 minutos. As sequências DP e T2 puderam ser adquiridas simultaneamente graças à programação do aparelho. A aquisição das imagens transversais e dorsais em DP e T2 demorou aproximadamente 27 minutos cada. A aquisição das imagens transversais e dorsais em T1 demorou aproximadamente 14 minutos cada. Portanto, o tempo de aquisição para o exame completo da cabeça nas sequências spin-eco T1, DP e T2 levou em média 74,5 minutos.

Esses achados demonstram que a técnica empregada é demorada. Tal resultado evidencia a necessidade dos cuidados de uma anestesia planejada e consideravelmente profunda para que o animal não se mova, apesar do tempo de exame e apesar do ruído alto emitido pelo apareIho em funcionamento, pois causaria perda da qualidade da imagem. Portanto, consideramos mais prudente a utilização da anestesia inalatória para a realização do exame "in vivo", levando em consideração ainda que os cães a serem encaminhados a esse exame não estariam em suas melhores condições de higidez, sendo dessa forma mais confiavelmente controlados. Sabendo que os aparelhos de anestesia inalatória comuns não são apropriados por motivos de segurança para utilização em salas de RM por serem confeccionados com materiais ferromagnéticos e que a utilização de longas traquéias com a finalidade de deixar o aparelho de anestesia longe do magnetismo levariam a um aumento incompatível do espaço morto a ser vencido pela respiração espontânea do animal, acreditamos piamente que para a realização do exame completo de RM da cabeça do cão em um animal vivo nesse tipo de tomógrafo 


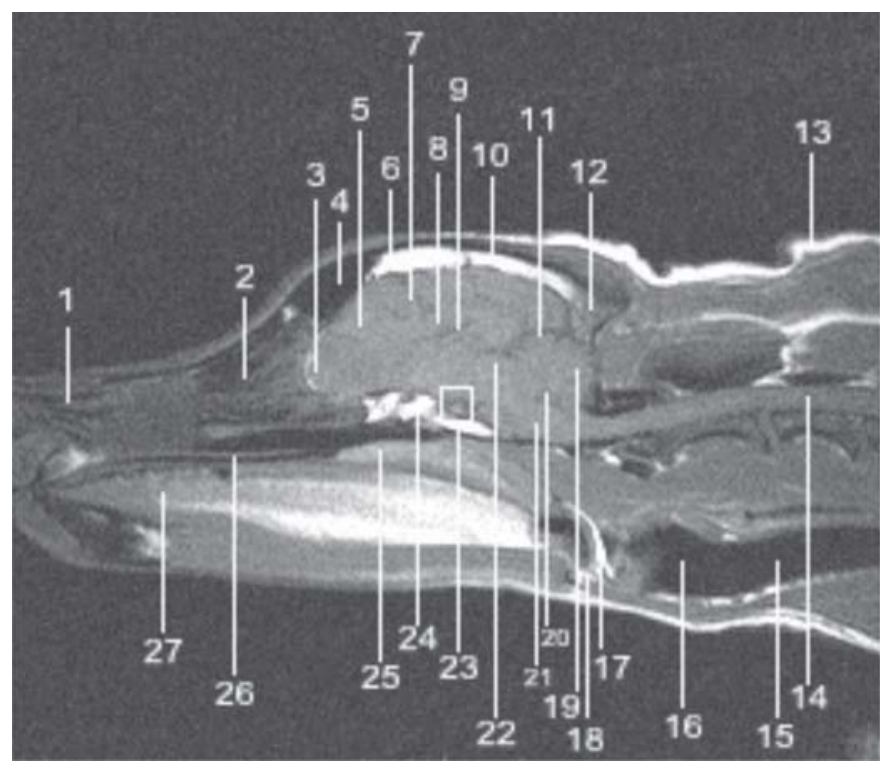

Fig.1. IRM em T1 da cabeça de cão, em corte sagital mediano, mostrando as estruturas identificadas: 1. Cavidade nasal, 2. Lâmina perpendicular do osso etmóide, 3. Bulbo olfatório, 4. Seio frontal, 5. Cérebro, 6. Osso frontal, 7. Sulco cruzado, 8. Corpo caloso, 9. Terceiro ventrículo, 10. Osso parietal, 11. Tentório ósseo do cerebelo, 12. Osso occipital, 13. Gordura subcutânea, 14. Medula espinhal, 15. Traqueia, 16. Laringe, 17. Epiglote, 18. Osso basiióide, 19. Cerebelo, 20. Quarto ventrículo, 21. Medula oblongata, 22. Ponte, 23. Hipófise, 24. Ossos esfenóides, 25. Palato mole, 26. Palato duro, 27. Língua.

de ressonância magnética seja necessário a prévia aquisição de um aparelho de anestesia inalatória próprio para utilização em sala de RM. Aparelhos mais modernos utilizam sequências mais rápidas como a fast spin-eco (Sage et al. 2006), o que reduz consideravelmente a duração do exame sem alterar a resolução da imagem, mas infelizmente não estavam disponíveis no aparelho onde foi realizado o estudo.
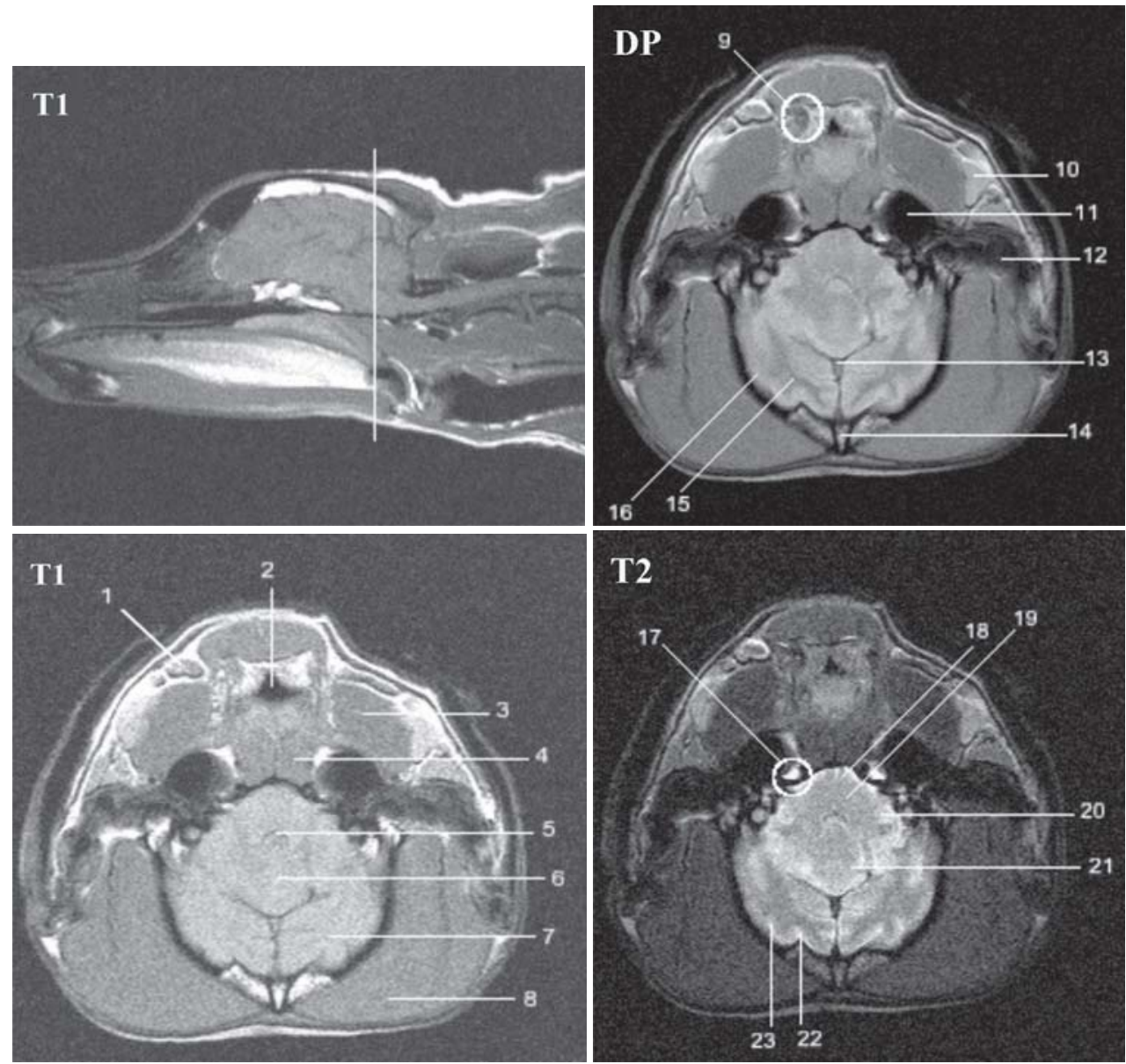

Fig.2. IRM em T1, DP e T2 da cabeça de cão, em corte transversal, mostrando as estruturas identificadas: 1. Linfonodos mandibulares, 2. Orofaringe, 3. Músculo digástrico, 4. Músculo longo da cabeça, 5. Quarto ventrículo, 6. Cerebelo, 7. Lobo occipital, 8. Músculo temporal, 9. Ceratoióide, 10. Glândula mandibular, 11. Bolha timpânica, 12. Meato acústico externo, 13. Tentório ósseo do cerebelo, 14. Crista sagital externa, 15. Substância branca, 16. Substância cinzenta, 17. Endolinfa no interior dos canais semicirculares, 18. Pirâmide, 19. Medula oblonga, 20. Paraflóculo, 21. Lóbulo ansiforme, 22. Sulco marginal, 23. Sulco ectomarginal. 

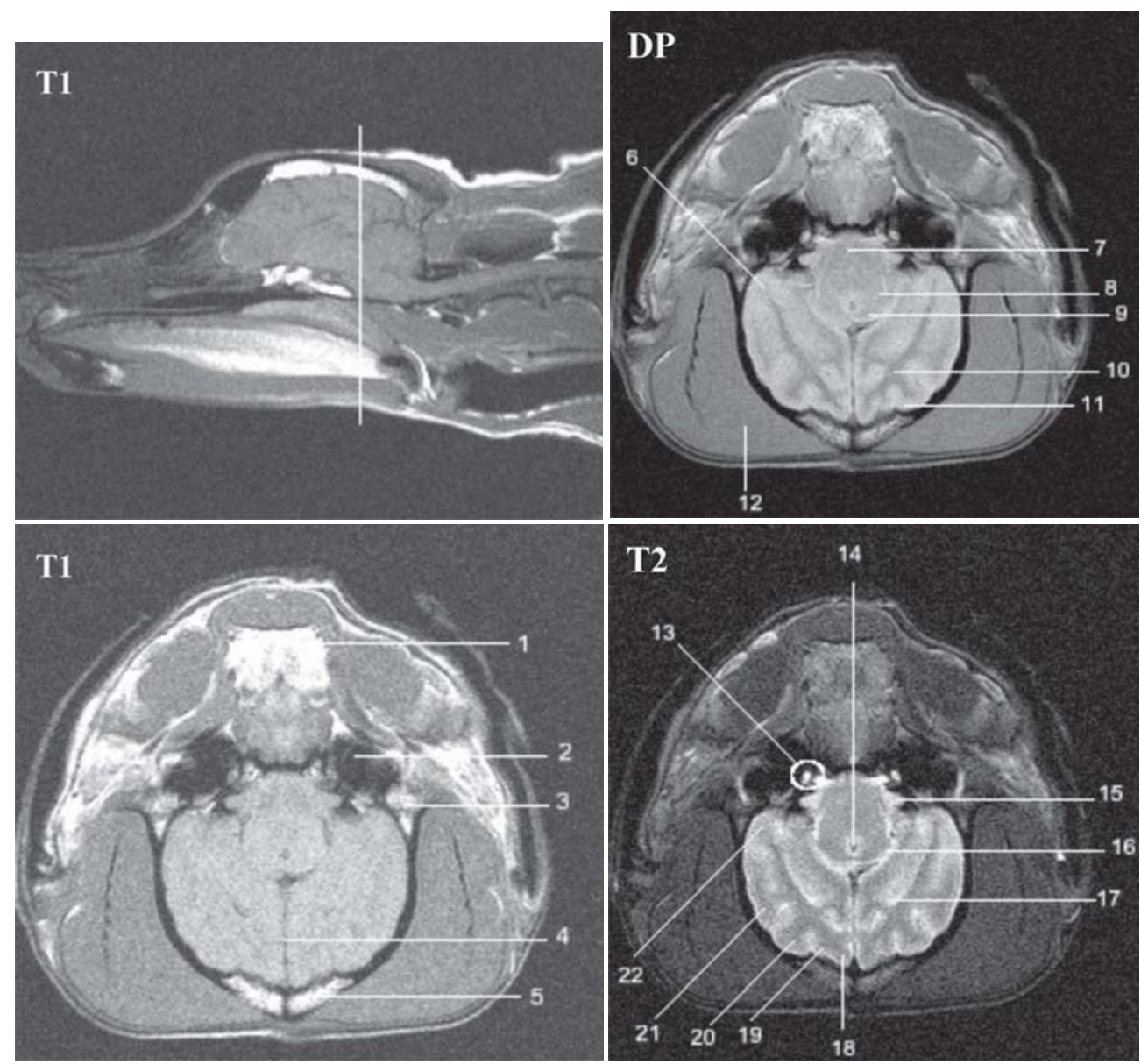

Fig.3. IRM em T1, DP e T2 da cabeça de cão, em corte transversal, mostrando as estruturas identificadas: 1. Língua, 2. Bolha timpânica, 3. Osso temporal, 4. Foice do cérebro, 5. Osso parietal, 6. Lobo temporal, 7. Tegmento do mesencéfalo, 8. Colículo caudal, 9. Colículo rostral, 10. Substância branca, 11. Substância cinzenta, 12. Músculo temporal, 13. Endolinfa no interior dos canais semicirculares, 14. Aqueduto mesencefálico, 15. Pedúnculo cerebelar, 16. Espaço subaracnóide, 17. Ventrículo lateral, 18. Giro marginal, 19. Giro ectomarginal, parte medial, 20. Giro ectomarginal, parte lateral, 21. Giro ectossilviano médio, 22. Giro ectossilviano caudal.

Esse estudo proporcionou imagens onde puderam ser identificadas diversas estruturas do sistema nervoso central, tais como: aderência intertalâmica, aqueduto mesencefálico, bulbo olfatório, cabeça do núcleo caudado, canal central, cápsula interna, cerebelo, cérebro, claustro, colículo caudal, colículo rostral, comissura rostral, coroa radiada, corpo amigdalóide, corpo caloso, corpo geniculado lateral, corpo geniculado medial, corpo mamilar, declive, espaço subaracnóide, esplênio do corpo caloso, fissura cerebral longitudinal, fissura de Sylvius, fissura longitudinal do cérebro, flóculo, forame interventricular, fórnix, fossa rombóidea, giro caudal de Sylvius, giro compósita rostral, giro do cíngulo, giro ectomarginal caudal, giro ectomarginal rostral, giro ectomarginal - parte lateral, giro ectomarginal - parte medial, giro ectossilviano caudal, giro ectossilviano médio, giro ectossilviano rostral, giro marginal, giro para-hipocampal, giro pós-cruzado, giro pré-cruzado, giro proreus, giro rostral de Sylvius, giro supra-silviano, hemisfério cerebelar, hemisfério cerebral, hipocampo, hipófise, lobo ansiforme, lobo frontal, lobo occipital, lobo parietal, lobo piriforme, lobo rostral, lobo temporal, medula espinhal, medula oblonga, nervo glossofaríngeo, nervo mandibular, nervo óptico, nervo trigêmeo, nervo vago, nervo vestibulococlear, núcleo caudado, paraflóculo, pedúnculo cerebelar, pirâmide, ponte, quarto ventrículo, quiasma óptico, substância branca, substância cinzenta, sulco ansiforme, sulco coronal, sulco cruzado, sulco ectomarginal, sulco ectossilviano, sulco marginal, sulco pré-silviano, sulco rinal, sulco rinal medial, sulco supra-silviano, tálamo, tegmento do mesencéfalo, terceiro ventrículo, tracto olfatório, tracto óptico, túber do vermis, ventrículo lateral e vermis. Achados esses que corroboram as citações de Kraft et al. (1989), Hudson et al. (1995), Assheuer \& Sager (1997) e Arencebia el al. (2005). Algumas dessas estruturas estão demonstradas nas figuras apresentadas (Fig.1-8).

Verificamos nessas imagens que o líquido cefalorraquidiano se apresentou hiperintenso em T2 e, portanto, apresentou alta conspicuidade em relação à cortical ós- 
sea da calota craniana que apresentou hipointensidade e o encéfalo que apresentou intensidade de sinal intermediária (Fig.3,4,6). Essas diferenças de contraste proporcionaram a pronta identificação dos giros e sulcos cerebrais em comparação com as sequências T1 e DP e facilitaram sobremaneira o estudo anatômico da região.

Essas características de contraste encontradas nesse estudo corroboram as explicações físicas para o fenômeno descritas por Lufkin (1999) que relata que tecidos com T2 longo possuem moléculas pequenas, como a água, que têm uma elevada frequência de movimento molecular (movimento Browniano), que faz com que a fase dos prótons seja mantida por um maior período de tempo. Como o sinal em T2 é medido no sentido transversal, quanto mais os prótons demorarem a relaxar, ou seja, quanto mais tempo demorarem a perder a fase, mais sinal esse tecido produzirá.

A imagem em densidade de prótons mostrou o melhor contraste entre a substância branca e a substância cinzenta do tecido encefálico (Fig.6). Nessa sequência as diferenças de contraste entre os tecidos somente se dão em decorrência das diferenças das densidades de prótons, ou seja, da quantidade de água livre nos tecidos, conforme esclarecido por Lufkin (1999). Portanto, sabendo que a densidade de prótons móveis na substância branca é $10 \%$ menor do que a da substância cinzenta, nossos achados concordam com o estudo de Kraft et al. (1989), Hudson et al. (1995), Assheuer \& Sager (1997) e Arencebia el al. (2005) que demonstra a menor intensidade de sinal da subtância branca em relação à substância cinzenta.

As imagens das estruturas encefálicas obtidas nesse estudo foram de excelente qualidade, porém atualmente, Kang et al. (2009) relataram imagens com melhor qualidade de contraste e resolução espacial do encéfalo com um tomográfo de RM com intensidade de campo magnético de 7 Tesla, principalmente das regiões da ponte e cerebelo.

Particularmente, a glândula pineal não foi observada nesse estudo e no estudo de Kang et al. (2009); esse fato
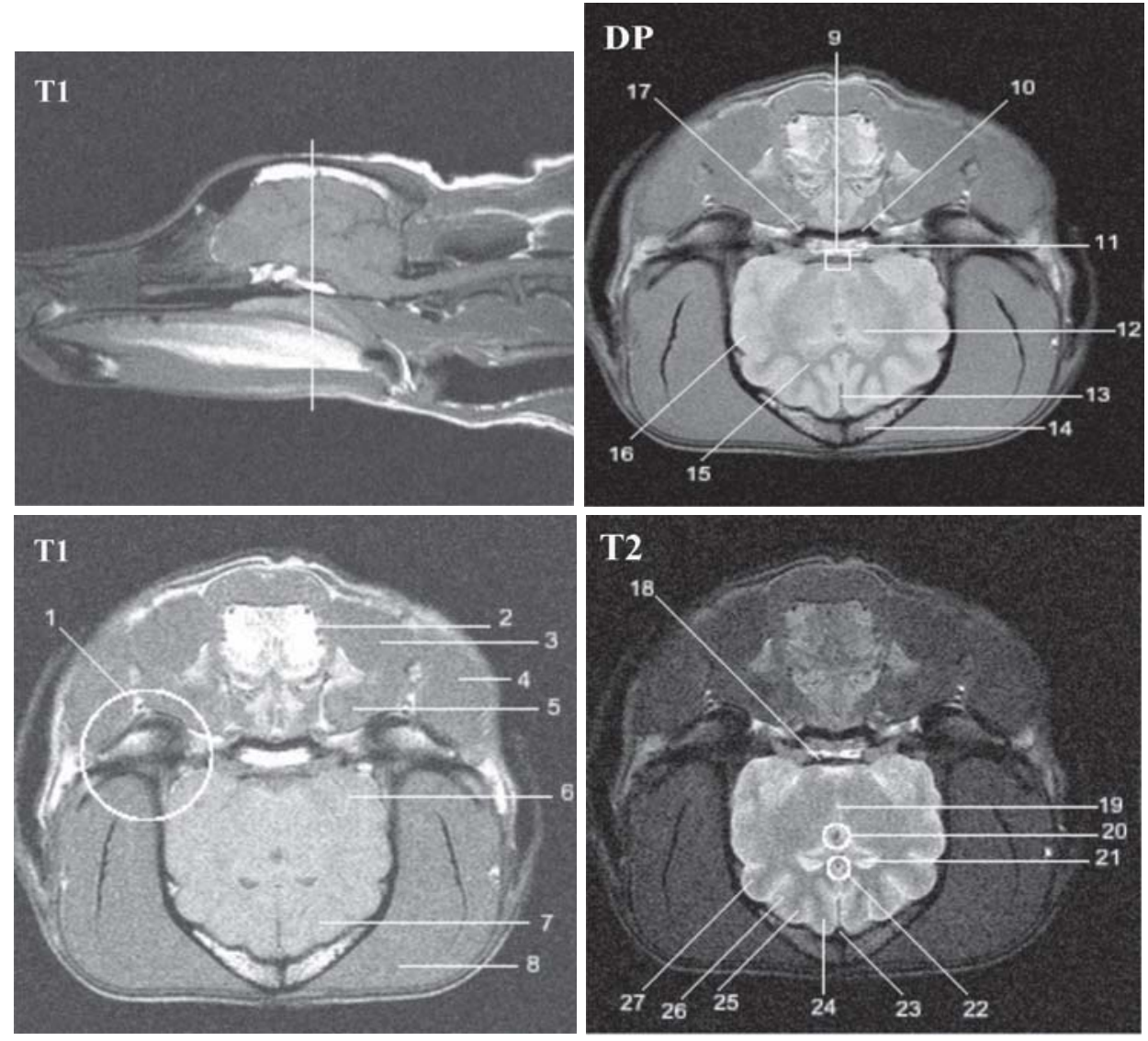

Fig.4. IRM em T1, DP e T2 da cabeça de cão, em corte transversal, mostrando as estruturas identificadas: 1. Articulação temporomandibular, 2. Língua, 3. Músculo digástrico, 4. Músculo masseter, 5. Músculo pterigóideo medial, 6. Lobo temporal, 7. Lobo parietal, 8. Músculo temporal, 9. Hipófise, 10. Nasofarínge, 11. Osso basisfenóide, 12. Núcleo caudado, 13. Foice do cérebro, 14. Osso parietal, 15. Substância branca, 16. Substância cinzenta, 17. Osso pterigóide, 18. Seio cavernoso, 19. Terceiro ventrículo, 20. Veia cerebral marginal, 21.Ventrículo lateral, 22. Veia do corpo caloso, 23. Seio sagital dorsal, 24. Giro marginal, 25. Giro ectomarginal, parte medial, 26. Giro ectomarginal, parte lateral, 27. Giro ectossilviano médio. 

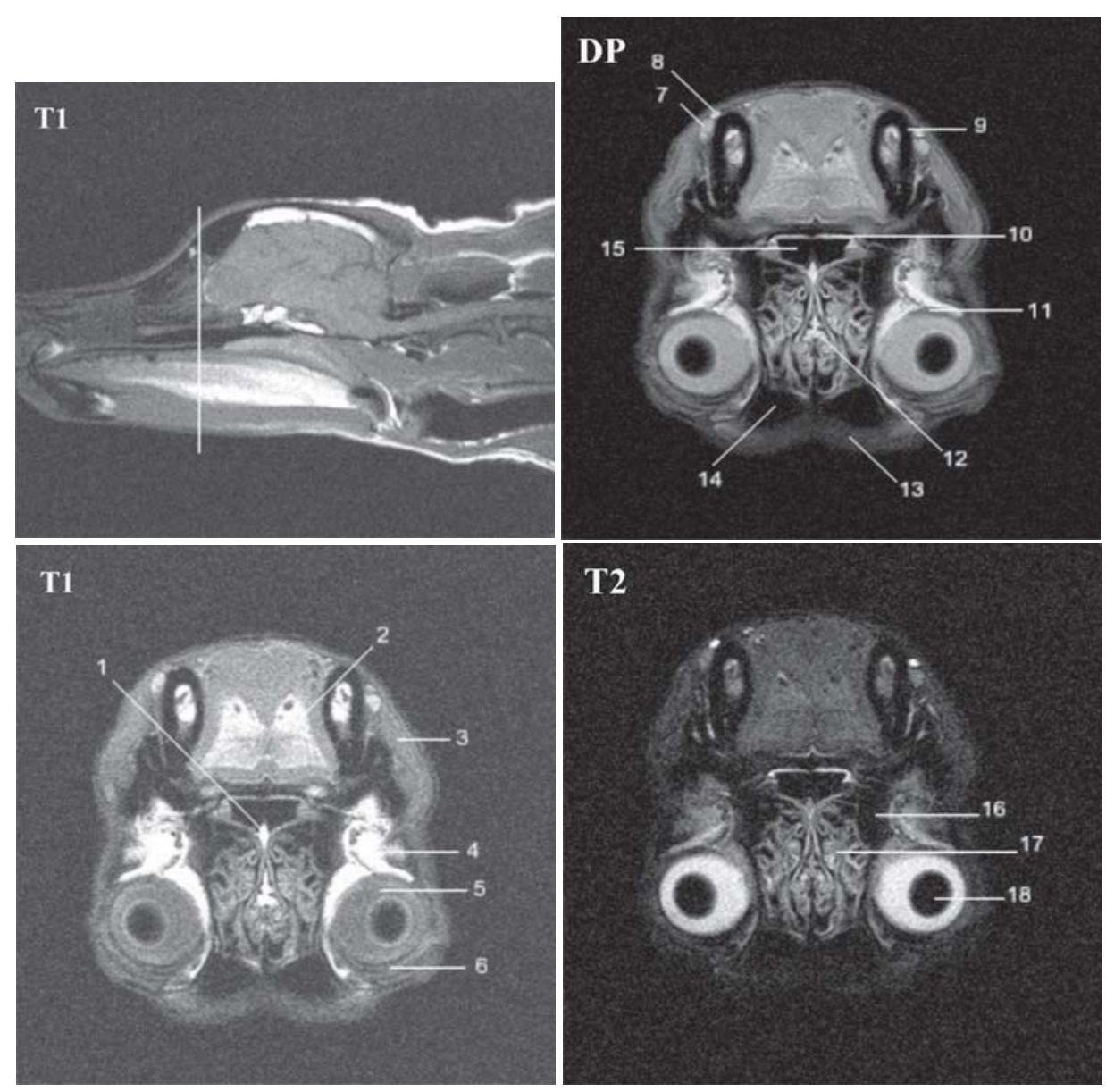

Fig.5. IRM em T1, DP e T2 da cabeça de cão, em corte transversal, mostrando as estruturas identificadas: 1. Vômer, 2. Língua, 3. Músculo orbicular da boca, 4. Arco zigomático, 5. Bulbo do olho, 6. Músculo orbicular do olho, 7. Glândulas bucais, 8. Veia labial inferior, 9. Mandíbula, 10. Osso palatino, 11. Músculo oblíquo ventral, 12. Osso etmóide, 13. Músculo frontal, 14. Seio frontal, 15. Meato nasofaríngeo, 16. Recesso maxilar, 17. Etmoturbinados, 18. Lente.

talvez possa ser explicado pelo seu tamanho diminuto (3mm x 1,5mm x 1mm) e localização (região caudal do terceiro ventrículo, na linha mediana, numa depressão entre o tálamo e os colículos rostrais), conforme descrito por Evans \& Miller (1993).

Esse estudo possibilitou a identificação de diversos músculos, como o músculo bucinador, músculo cleidocefálico - parte mastóidea, músculo digástrico, músculo esternoióideo, músculo frontal, músculo longo da cabeça, músculo masseter, músculo oblíquo caudal da cabeça, músculo oblíquo cranial da cabeça, músculo oblíquo ventral, músculo orbicular da boca, músculo orbicular do olho, músculo pterigóideo lateral, músculo pterigóideo medial, músculo reto lateral, músculo reto medial, músculo semi-espinhal da cabeça, músculo temporal, músculo ventricular, músculo vocal e músculos cervicais epaxiais. Alguns desses músculos estão demostrados nas figuras apresentadas (Fig.2,4,5,6,7,8). Os músculos apresentaram intensidade de sinal intermediária nas sequências utilizadas na seguinte ordem decrescente, T1>DP >T2, sendo que as imagens foram mais definidas em DP, sequência essa que demonstra as sutis diferenças na quantidade de prótons dos tecidos. Esses achados condizem com as informações da literatura (Kraft el al. 1989), porém as imagens produzidas nesse estudo proporcionaram a verificação dos mesmos cortes nas três sequências propostas.

As IRM demonstraram grande eficácia na apresentação das imagens desses músculos possibilitando a sua identificação. Diante desses achados concordamos com Hilário et al. (2000) sobre a importância do método para estabelecer a localização anatômica de lesões com envolvimento muscular, e vislumbramos a importância da sua aplicação na medicina veterinária.

Várias estruturas ósseas puderam ser identificadas nas imagens produzidas nesse estudo como o ângulo da mandíbula, ângulo frontal, arco zigomático, articulação temporomandibular, asa do atlas, asa do osso basisfenóide, asa do osso pré-esfenóide, atlas, bolha timpânica, borda frontal, borda sagital, cabeça da mandíbula, canal alar, canal óptico, ceratoióide, côndilo occipital, corpo do osso 
basisfenóide, corpo do osso pré-esfenóide, crista sagital externa, ectoturbinados, endoturbinados, etmoturbinados, foice do cérebro, hámulo pterigóide, lâmina cribriforme, lâmina perpendicular do osso etmóide, mandíbula, osso basiióide, osso basisfenóide, osso etmóide, osso frontal, osso occipital, osso palatino, osso parietal, osso préesfenóide, osso pterigóide, osso temporal, osso zigomático, ossos esfenóides, parte petrosa do osso temporal, processo coronóide do ramo da mandíbula, processo jugular do osso occipital, processo odontóide do áxis, processo zigomático do osso temporal, protuberância occipital externa, ramo da mandíbula, seio esfenoidal, seio frontal, tentório ósseo do cerebelo e vômer. Alguns desses ossos estão demonstrados nas figuras apresentadas (Fig.1-8).

A cortical óssea apresentou hipossinal nas três sequências avaliadas. Esse achado corrobora com os estudos anteriores de Kraft et al. (1989) e Hudson et al. (1995). Lufkin (1999) explica tal fato pela necessidade de interação dos prótons com os pulsos de RF para que seja possível a aquisição de um sinal e posterior formação da imagem. $\mathrm{Na}$ ausência de prótons, ou na presença desses em baixa densidade não há troca de energia e, portanto, não há coleta de sinal ocasionando uma falta de sinal na imagem reconstruída pelo computador, que é mostrada na tela pela cor preta e classificada à ressonância magnética como hipointensa. Além dessas características serem notadas nas corticais ósseas estudadas, também foram observadas nas estruturas que continham ar em seu interior como a traqueia, o seio frontal (Fig.1), a orofaringe, a bolha timpânica (Fig.2), o meato acústico externo (Fig.8), o meato nasofaríngeo (Fig.5) e o recesso maxilar (Fig.7) entre outras, além do ar ambiente ao redor do cadáver examinado.

A medular óssea apresentou sinal devido à presença de maior quantidade de prótons e de gordura e demonstrou variação da intensidade de sinal de acordo com a sequência utilizada. Em T1 apresentou hipersinal, em DP e em T2 apresentou sinal intermediário, sendo o sinal da DP > T2, acha-
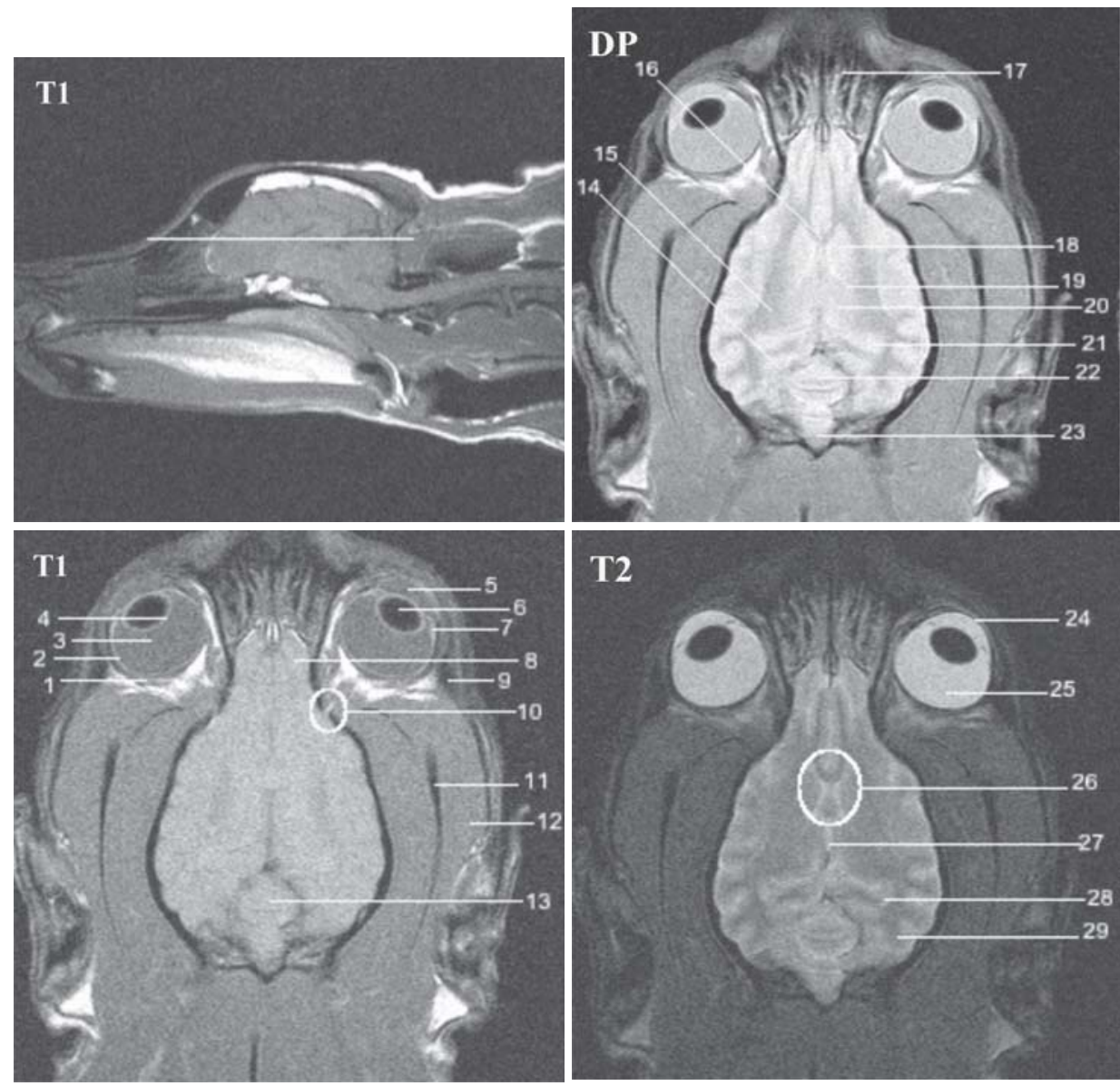

Fig.6. IRM em T1, DP e T2 da cabeça de cão, em corte dorsal ilustrado acima, mostrando as estruturas identificadas: 1. Esclera, 2. Retina, 3. Corpo vítreo, 4. Íris, 5. Câmara anterior, 6. Lente, 7. Corpo ciliar, 8. Bulbo olfatório, 9. Osso zigomático, 10. Nervo óptico, 11. Processo coronóide do ramo da mandíbula, 12. Músculo masseter, 13. Cerebelo, 14. Giro para-hipocampal, 15. Cápsula interna, 16. Comissura rostral, 17. Etmoturbinados, 18. Núcleo caudado, 19. Fórnix, 20. Tálamo, 21. Hipocampo, 22. Lobo rostral, 23. Declive, 24. Córnea, 25. Bulbo ocular, 26. Forame interventricular e ventrículos laterais, 27. Terceiro ventrículo, 28. Hipocampo, 29. Giro supra-silviano. 

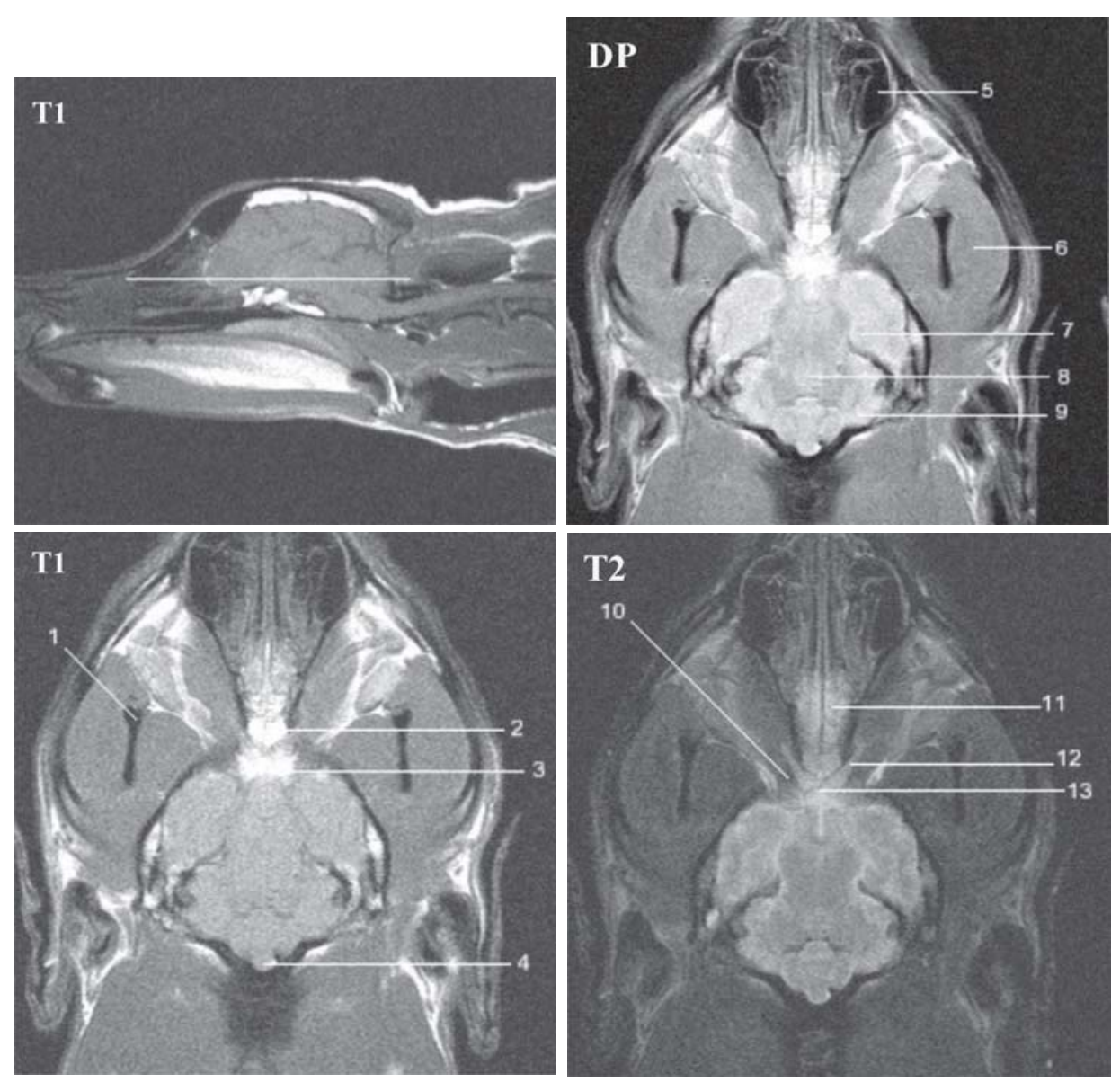

Fig.7. IRM em T1, DP e T2 da cabeça de cão, em corte dorsal, mostrando as estruturas identificadas: 1. Processo coronóide do ramo da mandíbula, 2. Osso pré-esfenóide, 3. Osso basisfenóide, 4. Pirâmide, 5. Recesso maxilar, 6. Músculo masseter, 7. Hipocampo, 8. Ponte, 9. Flóculo, 10. Canal óptico, 11. Bulbo olfatório, 12. Nervo óptico, 13. Quiasma óptico.

dos que corroboram as descrições de Arencebia et al. (2005).

Essas características de imagens encontradas nesse estudo corroboram as explicações físicas para o fenômeno descritas por Lufkin (1999) que relata que tecidos com T1 curto relaxam rapidamente, pois suas estruturas moleculares possuem frequência de oscilação próxima à fre-quência de Larmor do aparelho e, portanto, dissipam a energia absorvida pelo pulso de RF mais facilmente para o ambiente ao seu redor tornando-se susceptíveis ao próximo pulso de RF antes dos outros tecidos. Tecidos com essas características se apresentam brancos à ressonância magnética e são classificados como hiperintensos. Podese constatar isso observando o comportamento da gordura das regiões periocular, subcutânea, língua e regiões medulares dos ossos frontal, parietal, esfenóides e etmóide (Fig.5). Alves et al. (2007) comentam que do osso etmóide originam-se um complexo de pregas turbinadas (etmoturbinados), visibilizados nessa mesma figura.

Ciente disso entende-se as explicações de Hage \& Iwasaki (2009) de que um tecido que possui como característica um tempo de relação longo em $\mathrm{T} 1$, porque possui moléculas com frequência de oscilação muito acima ou muito abaixo da frequência de Larmor do aparelho, tem dificuldade para dissipar energia para o meio ao seu redor e, portanto, demora mais para relaxar não estando apto a responder a um novo pulso de RF, ou seja, o tecido com T1 longo fica refratário ao estímulo por mais tempo e, portanto, permanece na imagem sem sinal, ou seja, preto ou hipointenso. Pode-se constatar isso observando o comportamento do líquido cefalorraquidiano nos ventrículos laterais e sulcos cerebrais (Fig.4). Cabe lembrar aqui que não se devem confundir esses tecidos hipointensos que possuem T1 longo com os hipointensos devido a baixa densidade de prótons anteriormente citados.

A IRM normalmente não é utilizada para observação das estruturas ósseas do crânio uma vez que a tomografia computadorizada é considerada o padrão-ouro para esse fim, bem como a tomografia computadorizada de feixe cônico, para a região dentomaxilofacial (Roza et al. 2009), porém notou-se nas imagens produzidas que ela foi eficiente em demonstrar a região da medula óssea e, portanto, concordamos com Amaral et al. (2003) na indicação da IRM para detectar lesões ósseas nos estágios iniciais quando restritas à medula óssea. 
Foram visibilizadas nesse estudo diversas estruturas do bulbo ocular, como: câmara anterior, córnea, corpo ciliar, esclera, íris, lente, corpo vítreo e retina, além de estruturas associadas como a glândula lacrimal e o corpo adiposo da órbita. Algumas dessas estruturas estão demonstradas nas figuras apresentadas (Fig.5 e 6).

O humor vítreo e o humor aquoso apresentaram-se hiperintensos em T2 devido à grande quantidade de prótons na sua constituição (Fig.6). A lente a despeito do esperado apresentou-se hipointensa (preta) em T1, DP e T2 (Fig.5 e 6). Deve-se lembrar que apesar de rica em água, e, portanto, em hidrogênio, a lente apresenta uma estrutura sólida e, portanto, não é capaz de apresentar vibração de suas moléculas. Pode-se fazer uma correlação com um cubo de gelo, pois apesar de rico em água não está apto a emitir sinal de ressonância magnética por estar em estado sólido.

A capacidade de identificação das estruturas do bulbo ocular, associada à vantagem dos cortes multiplanares com ausência de sobreposição de estruturas tornam a IRM um método excelente para visibilizar as estruturas dos olhos. Porém, concordamos com Morgan et al. (1994) e Dennis (2000) que sugerem que sua utilização deva ser reservada para os casos onde a ultrassonografia e a radiologia não obtiveram sucesso na obtenção do diagnóstico e não há suspeita de corpo estranho ferromagnético que poderia ser atraído pelo equipamento e causar maiores lesões.

O estudo permitiu a visibilização de estruturas do aparelho respiratório como: cartilagem aritenóide, cartilagem tireóide, cavidade nasal, coanas, epiglote, laringe, meato nasofaríngeo, nasofaringe, orofaringe, recesso maxilar, tonsila palatina e traqueia. Observou-se nesse estudo que a epiglote apresentou hiperintensidade em T1 (Fig.1), achado que concorda com o descrito por Vázquez et al. (1998) e que explica tal fato devido a epiglote ser uma estrutura cartilagínea de natureza elástica, rica em fibra colágena tipo II, com alta densidade de prótons.

Estruturas do aparelho digestório também foram detectadas como a glândula mandibular (Fig.1), a glândula parótida (Fig.8), a glândula zigomática, as glândulas bucais (Fig.5), a língua, o palato duro, o palato mole (Fig.1),
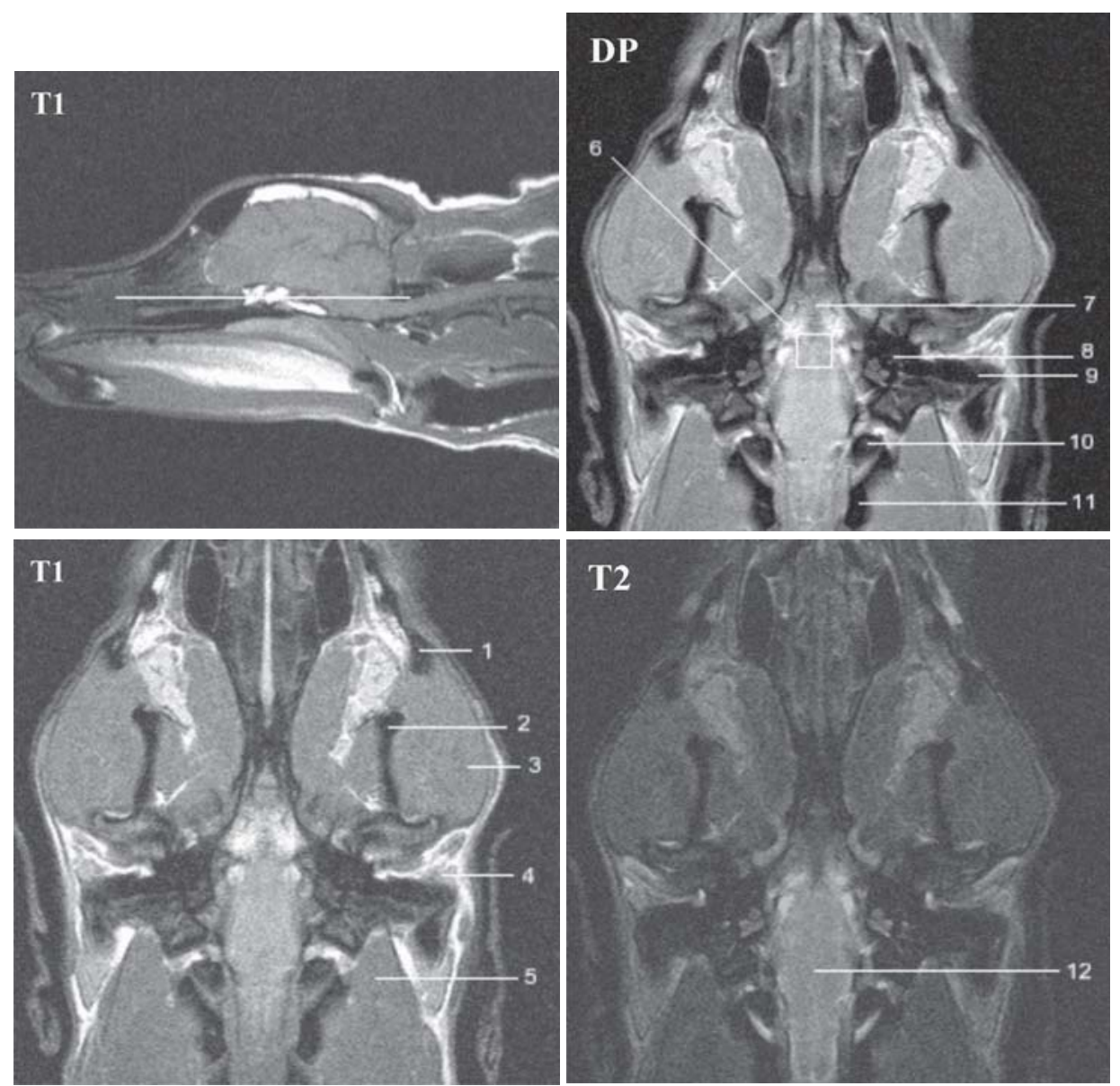

Fig.8. IRM em T1, DP e T2 da cabeça de cão, em corte dorsal, mostrando as estruturas identificadas: 1. Arco zigomático, 2. Ramo da mandíbula, 3. Músculo masseter, 4. Glândula parótida, 5. Músculo longo da cabeça, 6. Hipófise, 7. Quiasma óptico, 8. Bolha timpânica, 9. Meato acústico externo, 10. Côndilo occipital, 11. Atlas, 12. Medula oblonga. 
o primeiro dente molar inferior, o quarto dente pré-molar superior, o terceiro dente molar e o vestíbulo oral.

Esse estudo permitiu a visibilização de algumas estruturas vasculares da cabeça, como: artéria carótida comum, artéria carótida interna, artéria lingual, artéria palatina ascendente, artéria vertebral, seio cavernoso, seio sagital dorsal, seio transverso, veia cerebral magna, veia do corpo caloso, veia facial, veia labial inferior e veia lingual. Algumas dessas estruturas estão demonstradas(Fig.4). Apesar da possibilidade de visibilização dos vasos nesse estudo, Spotti et al. (2001) indicam a angiorressonância magnética como método mais eficaz na visibilização de estruturas vasculares, na qual são realizadas sequências de gradiente eco, onde o sangue circulante age como contraste e, através do fluxo sanguíneo, são obtidos sinais que se transformam em imagem. A forma de reconstrução tridimensional espacial da angiorressonância magnética subtrai a imagem do tecido estacionário, possibilitando uma melhor visibilização do território vascular.

Os linfonodos submandibulares também foram identificados nesses estudos (Fig.2) e apresentaram padrão hipointenso em relação à gordura adjacente em T1 e T2, concordando com os achados de Kneissl \& Probst (2006). Esses autores sugerem que pode ser necessário a utilização de sequências com alto poder de resolução, como a gradiente eco 3D com reconstrução multiplanar, para verificar a suspeita de linfonodos anormais.

A endolinfa no interior dos canais semicirculares, em T2, foi visibilizada apresentando hipersinal (branca) e contrastou com o labirinto ósseo ao redor que não apresentou sinal (preto) (Fig. 2, 3). Diante desse achado concordamos com Garosi et al. (2003) que a capacidade de identificação por meio da IRM da endolinfa na orelha interna do cão normal é uma das grandes vantagens dessa modalidade de imagem. Tal achado permite que se diferencie uma oreIha interna normal de uma em fase crônica de inflamação, onde a obliteração fibrosa dos espaços contendo linfa causa perda desse sinal. Diante disso vislumbra-se que quando a IRM for uma realidade na medicina veterinária nacional realizar-se-á com maior facilidade a diferenciação entre desordens vestibulares de origem central e periférica.

Nesse estudo as sequências T1, DP e T2 se mostraram complementares no estudo da anatomia da cabeça do cão, discordando de Kraft et al. (1989), Morgan et al. (1994) e Vázquez et al. (1998), os quais citam T1 como a sequência de escolha para os estudos anatômicos.

\section{CONCLUSÕES}

As IRM adquiridas nas sequências T1, DP e T2 foram complementares para o estudo dos aspectos anatômicos da cabeça de cães, demonstrando-os com riqueza de detalhes.

O tempo requerido para o exame completo da cabeça é compatível para uso em animais vivos, desde que devidamente anestesiados e controlados.

Os resultados obtidos por esse trabalho são reproduzíveis e dessa forma abrem caminho para o estudo de animais vivos e para o início da investigação de doenças, principalmente as de origem neurológica, visto ser essa técnica excelente para a visibilização do encéfalo.

Agradecimentos.- À Fundação de Amparo à Pesquisa do Estado de São Paulo (FAPESP) pelo financiamento do projeto (Proc.02/10.4840). À Coordenação de Aperfeiçoamento de Pessoal de Nível Superior (CAPES) pela bolsa de doutorado da primeira autora. Aos colaboradores Jean Louis Shinohara, Carla Aparecida Batista Lorigados e Hildebrando Gomes Benedicto.

\section{REFERÊNCIAS}

Alves F.R., Santos T.C., Freiberger S., Ambrósio C.E. \& Miglino M.A. 2007. Dinâmica dos precursores celulares do epitélio olfatório de cães sem raça definida: um estudo imunohistoquímico e ultraestrutural. Pesq. Vet. Bras. 27:388-392.

Amaral L., Chiurciu M., Almeida J.R., Ferreira N.F., Mendonça R. \& Lima S.S. 2003. MR imaging evaluation of lesions of the cranial vault. Arq. Neuro-Psiquiatr. 61:521-532.

Arencibia A., Rivero M.A., Gil F., Ramírez, J.A., Orbera J.A., Ramírez G. \& Vásquez J. M. 2005. Anatomy of the cranioencephalic structures of the camel (Camelus dromedarius L.) by imaging techniques: a magnetic resonance imaging study. Anat. Histol. Embryol. 34:52-55.

Arencibia A., Vázquez. M., Ramírez J. A., Ramírez G., Vilar J.M., Rivero M.A., Alayon S., \& Gil F. 2001. Magnetic resonance imaging of the normal equine brain. Vet. Radiol. Ultrasound 42:405-408.

Assheuer J. \& Sager M. 1997. Mri and CT Atlas of the Dog. Blackwell Science, Oxford, p.482.

Blink A., Berkefeld J. \& Zanella F. 2009. Anatomie der Schädelbasis und Hirnnerven in der Schnittbildgebung. Radiologe 49:584-597.

Chaffin M.K., Walker M.A., McArthur N.H., Perris E.E. \& Matthews N.S. 1997. Magnetic resonance imaging of the brain of normal neonatal foals. Vet. Radiol. Ultrasound 38:102-111.

Dennis R. 2000. Use of magnetic resonance imaging for the investigation of orbital disease in small animals. JSAP 41:145-155.

De Rycke L.M., Saunders J.H., Gielen I.M., van Bree H.J. \& Simoens P.J. 2003. Magnetic resonance imaging, computed tomography, and cross-sectional views of the anatomy of normal nasal cavities and paranasal sinuses in mesaticephalic dogs. Am. J. Vet. Res. 63:10931098.

Desal H.A., Auffray-Calvier E., Guillon B., Toulgoat F., Madoz A., De Kersaint-Gilly A. \& Pasco-Papon A. 2004. Imagerie des accidents vasculaires cerebraux em urgence. J. Neuroradiol. 31:327-333.

Evans H.E. \& Miller M.E. 1993. Evans-Miller's Anatomy of the Dog. 3rd ed. W.B. Saunders, Philadelphia, p.1113.

Feeney D.A., Fletcher T.F. \& Hardy R.M. 1991. Atlas of Correlative Imaging Anatomy of the Normal Dog: Ultrasound and Computed Tomography. W.B. Saunders, Philadelphia, p.383.

Fonseca-Pinto A.C.B.C., Hage M.C.F.N.S. \& Burguese L.F. 2008. Tomografia computadorizada e ressonância magnética, p.694-718. In: Santos, M.M. \& Fragata, F.S. Emergência e terapia intensiva veterinária em pequenos animais. Ed. Roca, São Paulo.

Garosi L.S., Dennis R. \& Schwarz T. 2005. Review of diagnostic imaging of the ear diseases in the dog and cat. Vet. Radiol. Ultrasound 44:137146.

Hage M.C.F.N.S. \& Iwasaki M. 2009. Imagem por ressonância magnética: princípios básicos. Ciência Rural 39:1275-1283.

Hermie M., Leal P.R., Salaris S.F., Froment J.C. \& Sindou M. 2009. Imagerie anatomique des nerfs crâniens. Neurochirurgie 55:162-173.

Hilário M.O.E., Yamashita H., Lutti D., Len C., Teresi M.T. \& Lederman H. 2000. Juvenile idiopathic inflamatory myopathies: the value of magnetic resonance imaging in the detection of muscle involvement. São Paulo Med. J. 118:35-40. 
Hudson L.C., Cauzinille L., Kornegay J.N. \& Tompkins M.B. 1995. Magnetic-resonance-imaging of the normal feline brain. Vet. Radiol. Ultrasound 36:267-275.

Kang B., Ko K., Jang D, Han J., Lim C., Park C., Yoo J., Kim J., Jung D., Kim Y., Woo E., Cho Z. \& Park H. 2009. Magnetic resonance imaging of the canine brain at $7 \mathrm{~T}$. Vet. Radiol. Ultrasound 50:615-621.

Kneissl S. \& Probst A. 2006. Magnetic resonance imaging features of presumed normal head and neck lymph nodes in dogs. Vet. Radiol. Ultrasound 47:538-541.

Kraft S.L., Gavin P.R., Dehaan C., Moore M., Wendling L.R. \& Leathers C.W. 1997. Retrospective review of 50 canine intracranial tumors evaluated by magnetic resonance imaging. J. Vet. Intern. Med. 11:218-255.

Kraft S.L., Gavin, P.R., Wendling L.R. \& Reddy V.K. 1989. Canine brain anatomy on magnetic resonance images. Vet. Radiol. 30:147158.

Lufkin R.L. 1999. Manual de Ressonância Magnética. Guanabara Koogan, Rio de Janeiro, p.338.

Marino L., Sudheimer K.D., Murphy T.L., Davis K.K., Pabst D.A., McLellan W.A., Rilling J.K. \& Johnson J.I. 2001. Anatomy and three-dimensional reconstructions of the brain of a Bottlenose Dolphin (Tursiops trucatus) from magnetic resonance images. Anat. Rec. 264:397-414.

Morgan R.V., Daniel G.B. \& Donnel R.L. 1994. Magnetic resonance imaging of the normal eye and orbit of the dog and cat. Vet. Radiol. Ultrasound 35:102-108.
Mullins M.E. 2006. Modern emergent stroke imaging: pearls, protocols and pitfall. Radiol. Clin. North Am. 44:41-62.

Oishi K., Zilles K., Amunts K., Faria A., Jiang H., Li X., Akhter K., Hua K., Woods R., Toga A.W., Pike G.P., Rosa-Neto P., Evans A., Zhang J., Huang H., Miller M.I., van Zijl P.C.M., Mazziotta J. \& Mori S. 2008. Human brain white matter atlas: identification and assignment of common anatomical structures in superficial white matter. Neuroimage 43:447-457.

Platt S.R. \& Garosi L. 2003. Canine cerebrovascular disease: Do dogs have strokes? J. Am. Anim. Hosp. Assoc. 39:337-342.

Roza M.R., Silva L.A.F., Januário A.L., Barriviera M., Oliveira A.C.S. \& Fioravanti M.C.S. 2009. Tomografia computadorizada de feixe cônico na odontologia veterinária: descrição e padronização da técnica. Pesq. Vet. Bras. 26:617-624.

Sage J.E., Samii V.F., Abramson C.J., Green E.M., Smith M. \& Dingus C. 2006. Comparison of conventional spin-echo and fast spin-echo magnetic resonance imaging in the canine brain. Vet. Radiol. Ultrasound 47:249-253.

Schaller O. 1999. Nomenclatura Anatômica Veterinária llustrada. Editora Manole, São Paulo, p.614.

Spotti A.R., Lima E.G., Santos M.L.T. \& Magalhães A.C.A. 2001. Angiografia pela ressonância magnética nos aneurismas intracranianos. Arq. Neuro-Psiquiatr. 59(2B):384-389.

Vázquez J.M., Arencibia A., Gil F., Ramírez J.A., González N., Sosa C.D. \& Jaber J.R. 1998. Magnetic resonance imaging of the normal canine larynx. Anat. Histol. Embryol. 27:263-270. 